\title{
Green synthesis and catalytic application of curcumin stabilized silver nanoparticles
}

\author{
A D VERMA, N JAIN, S K SINGHA ${ }^{\mathrm{a}}$, M A QURAISHI and I SINHA* \\ Department of Chemistry, Indian Institute of Technology (Banaras Hindu University), Varanasi, Uttar Pradesh \\ 221005 , India \\ a Present Address: Indian School of Mines, Dhanbad, India \\ e-mail: isinha.apc@iitbhu.ac.in
}

MS received 3 August 2016; revised 27 September 2016; accepted 27 September 2016

\begin{abstract}
An ultrasonication based green synthesis approach was used to prepare curcumin-stabilized silver nanoparticles (c-AgNPs). Nanoparticles thus obtained were characterized by UV-Visible spectroscopy, X-ray diffraction (XRD), Transmission electron microscopy (TEM) and Fourier transform infrared spectroscopy (FTIR). Two different size distributions of c-AgNPs were obtained by changing the ratio of curcumin to silver salt precursor. These c-AgNPs were used as catalysts in the catalytic reduction of $p$-nitrophenol to $p$-aminophenol. The c-AgNPs with narrower size distribution exhibited better catalytic activity as well as lower activation energy. Variation of apparent rate constant with the reactant concentration agreed with the LangmuirHinshelwood (LH) model. Consequently, the surface rate constant related to the rate-determining step and the respective adsorption constants of $p$-nitrophenol and of borohydride were determined as per this model.
\end{abstract}

Keywords. Green synthesis; curcumin stabilized silver nanoparticles; p-nitrophenol; catalytic kinetics; Langmuir-Hinshelwood model.

\section{Introduction}

Noble metal nanoparticles have many applications in optical, electronic, chemical, biological, and medical fields due to their characteristic properties. ${ }^{1-7}$ While there are many techniques for obtaining these nanoparticles, for environmentally friendly synthesis protocols, many researchers have turned toward natural substances such as vitamins, sugars, plant extracts, biodegradable polymers, and microorganisms as reducing and capping agents. Plant based materials seem to be the best candidates and they are suitable for large-scale 'biosynthesis' of nanoparticles. ${ }^{8,9}$ The key or active agent in some of these synthesis are believed to be polyphenols, like in the case of tea, wine and winery waste, red grape pomace. ${ }^{10,11}$ Similarly, curcumin, the main polyphenol in turmeric has recently been used as a stabilizing and reducing agent in synthesis of $\mathrm{Au}$ and Ag nanoparticles. ${ }^{12-14}$ To the best of our knowledge, there is no study on whether curcumin stabilization of such noble metal nanoparticles has any effect on their catalytic properties.

Keeping the foregoing in perspective, this communication presents a detailed study on the catalytic activity of curcumin stabilized silver nanoparticles (c-AgNPs).

*For correspondence
The catalytic efficiency of curcumin stabilized nanoparticles was tested by using them as catalysts in the model reaction of reduction of $p$-nitrophenol (Nip) to $p$-aminophenol (AP). This is a well-known model reaction which occurs in ambient conditions and without any side reactions. Using such a standard reaction could make possible the study of the catalytic kinetics from the perspective of elucidating the mechanistic details. Furthermore, this reaction results in the removal of nitrophenols, which are regarded as one of the most widespread environmental pollutants, while nitro to amino conversion process is of high industrial relevance such as for production of aniline, paracetamol, etc. ${ }^{15}$ It is worthwhile to mention here that there are only a few reports in literature on reduction of Nip over AgNPs but with little or no kinetic studies. ${ }^{16,17}$

In the present study, therefore, the first step was the preparation of c-AgNPs by an ultrasound assisted synthesis protocol. We explored ultrasound assisted or sonochemical reduction route for this synthesis to overcome the general disadvantage of longer reaction time, ${ }^{15}$ generally associated with such green synthesis. In the sonochemical technique acoustic cavitation due to high intensity ultrasonic irradiation of water leads to formation of highly reactive $\mathrm{H} \bullet$ and $\mathrm{OH} \bullet$ radicals. Sonochemically generated $\mathrm{H} \bullet$ radicals or secondary radical species from organic additives in the 
reaction mixture are considered to act as reductant in such metal nanoparticle synthesis. ${ }^{18} \mathrm{c}$-AgNPs with two different size distributions were prepared by taking different amounts of curcumin in the synthesis. Next, we applied these two c-AgNPs samples as catalysts in the model reduction reaction of Nip to AP. These c-AgNPs exhibit excellent catalytic activity for Nip reduction. At a fixed reactant concentration, the effect of temperature on the kinetics was investigated.

Recent publications from Ballauff's and Meijboom's groups have shown that such data for some catalysts can be modeled by the Langmuir-Hinshelwood (LH) mechanism. ${ }^{19-24}$ The LH model implies that first both reactants adsorb on the surface of the catalyst. In the rate determining step, the metal nanocatalyst surface acts as the electron conduit for transfer of electrons from the donor $\mathrm{BH}_{4}^{-}$to the nitrophenolate ions for its reduction. Therefore, detailed studies on the effect of reactant concentrations on catalytic kinetics were also carried out and the kinetic data generated were subjected to the LH model to understand the catalytic mechanism better.

\section{Experimental}

\subsection{Synthesis of curcumin stabilized AgNPs (c-AgNPs)}

Materials used for the synthesis of c-AgNPs were $\mathrm{AgNO}_{3}$ (Merck), Curcumin (Sigma Aldrich) and $\mathrm{NaOH}$ (Merck). All reagents used were of analytical reagent grade and used without further purification. Curcumin solutions of $0.01 \mathrm{M}$ and $0.001 \mathrm{M}$ concentration were prepared by dissolving suitable amount of curcumin in $2 \mathrm{~mL}$ of $10 \mathrm{mM}$ aqueous $\mathrm{NaOH}$ solution and then the volume was made up to $10 \mathrm{~mL}$ by water. Next, $5 \mathrm{~mL}$ each of these freshly prepared curcumin solutions were added to $0.05 \mathrm{mmol}$ of $\mathrm{AgNO}_{3}$ taken in $40 \mathrm{~mL}$ water and subjected to ultrasonication for $5 \mathrm{~min}$ (with $25 \mathrm{~s}$ 'on' and $10 \mathrm{~s}$ 'off' cycles). On completion of this treatment the reaction mixture turned from yellowish red to greenish yellow which indicated the formation of c-AgNPs. Hereafter, we denote the c-AgNPs sample prepared as $\mathrm{S} 1$ when the precursor silver salt to curcumin ratio was taken as $1: 1$ and as $\mathrm{S} 2$ when this ratio was taken to be 1:0.1.

\section{2 p-nitrophenol reduction}

For this purpose, $0.1 \mathrm{~mL}$ of $1.2 \times 10^{-3} \mathrm{M}$ Nip was diluted by adding $1.85 \mathrm{~mL}$ water and taken in a standard quartz cuvette of $1-\mathrm{cm}$ path length. To this $50 \mu \mathrm{L}$ of aqueous $0.1 \mathrm{M} \mathrm{NaBH}_{4}$ and $40 \mu \mathrm{L}$ of the as-prepared
c-AgNPs sol solution were added. The absorption spectrum of this reaction mixture was recorded after every two minutes interval. The same procedure was repeated to monitor the kinetics of the c-AgNPs catalyzed reaction at four temperatures 297, 301, 305 and $309 \mathrm{~K}$. The reaction kinetics was also studied by varying the reactant concentrations at constant temperature $301 \mathrm{~K}$. To study the effect of $\mathrm{NaBH}_{4}$ concentration on the reaction kinetics, $0.1 \mathrm{~mL}$ of $1.2 \times 10^{-3} \mathrm{M}$ Nip, diluted by adding $1.85 \mathrm{~mL}$ water, was taken. To this $50 \mu \mathrm{L}$ of aqueous $\mathrm{NaBH}_{4}$ solution of different concentrations $(0.05,0.1$, $0.2,0.3,0.4 \mathrm{M}$ ) were added. Then, $40 \mu \mathrm{L}$ of the asprepared c-AgNPs sol sample was added. Similarly, the effect of increasing Nip concentration on the kinetics of the reaction was also investigated. Here, the concentration of $\mathrm{NaBH}_{4}$ was fixed at $0.1 \mathrm{M}$ while that of Nip concentration was varied as $0.6,1.2,1.8,2.4,3.0 \times 10^{-3} \mathrm{M}$.

\section{Results and Discussion}

\subsection{Characterization of c-AgNPs}

Figure 1 shows the UV-Visible absorbance spectrum of the as-prepared (diluted) c-AgNPs sols, S1 and S2. For both $\mathrm{S} 1$ and $\mathrm{S} 2$, the maximum UV-Visible absorbance was found to be $408 \mathrm{~nm}$ in agreement with the wellknown LSPR absorbance of aqueous dispersion of silver nanoparticles. ${ }^{17,25}$ The X-ray diffraction pattern of the nanoparticle powder samples S1 and S2 (Figure 2) show five peaks at $38.18^{\circ}, 44.40^{\circ}, 64.66^{\circ}, 77.07^{\circ}$ and $81.25^{\circ}$. As per XRD data reported for silver in JCPDSICDD (card no. 87-0720), these correspond to the [111], [200], [220], [311] and [222] planes of fcc silver.

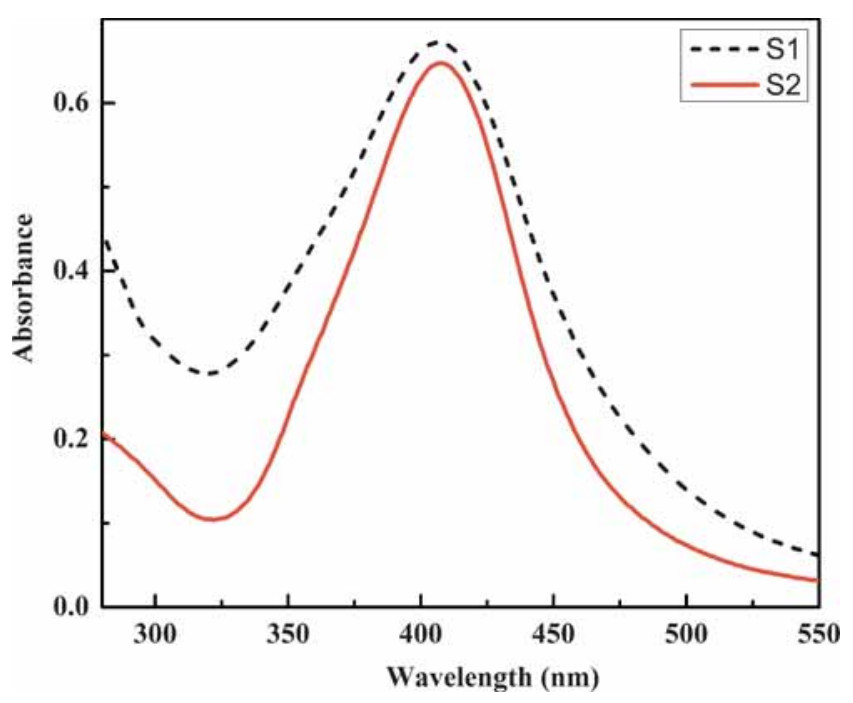

Figure 1. The UV-VIS absorption spectra of aqueous dispersion of samples S1 (10 times diluted) and S2 (as prepared) c-AgNPs sols. 


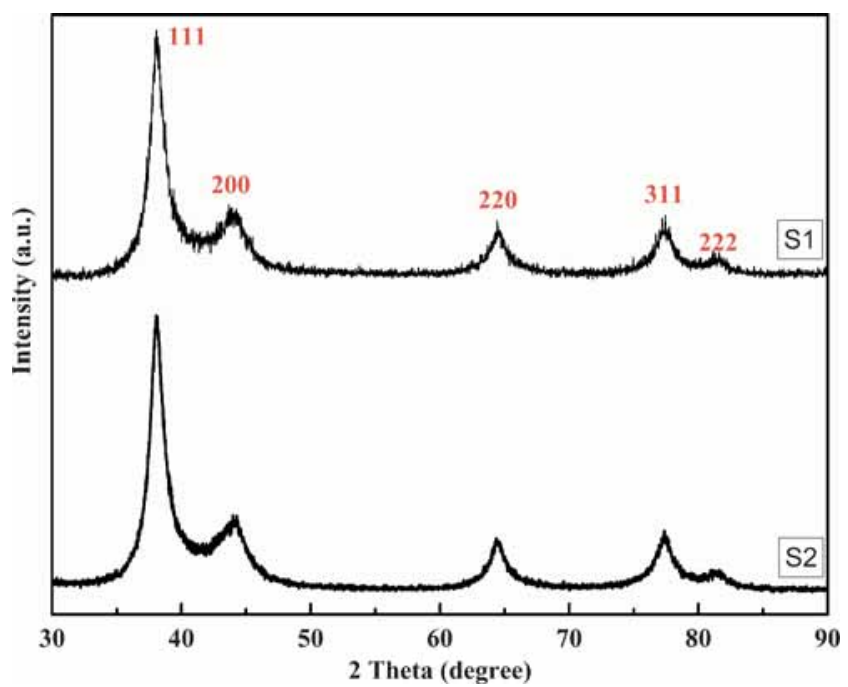

Figure 2. X-ray powder diffraction pattern of S1 and S2 c-AgNPs.

A typical TEM micrograph and the respective particle size distributions of S1 and S2 samples are shown in Figure 3. The c-AgNPs of sample $\mathrm{S} 1$ are found to be uniformly spherical in shape with an average size of $\sim 17 \mathrm{~nm}$ as indicated by the size distribution histogram (Figure 3b). On the other hand, c-AgNPs of sample S2 have narrower size distribution as well as finer average particle size $(12 \mathrm{~nm})$. The size of nanoparticles was calculated from the TEM micrographs. The average size was calculated by measuring approximately 300 nanoparticles. For analyzing the interactions between c-AgNPs and functional groups associated with curcumin, the FTIR spectra of both pure curcumin and solid powder c-AgNPs (S2) sample (Figures 4a and 4b) were recorded in the range of $4000-400 \mathrm{~cm}^{-1}$ in the diffuse reflectance mode operating at resolution of $1 \mathrm{~cm}^{-1}$ using Perkin Elmer Spectrum 100 FT-IR spectrometer. To obtain a good signal to noise ratio, 16 scans of AgNPs were taken using $\mathrm{KBr}$ pellets. As can be seen from a comparison of the FTIR spectra of curcumin and c-AgNPs, the bands at $2922 \mathrm{~cm}^{-1}, 1462 \mathrm{~cm}^{-1}, 1030$ $\mathrm{cm}^{-1}$ and $959 \mathrm{~cm}^{-1}$ that appear in c-AgNPs are due to vibrations of aliphatic $\mathrm{C}-\mathrm{H}$ stretches and mixed vibrations of $\mathrm{CH}_{3}$, aromatic $\mathrm{CCC}$ and $\mathrm{CCH}$ of curcumin. This confirms that curcumin acts as a capping agent. ${ }^{12}$

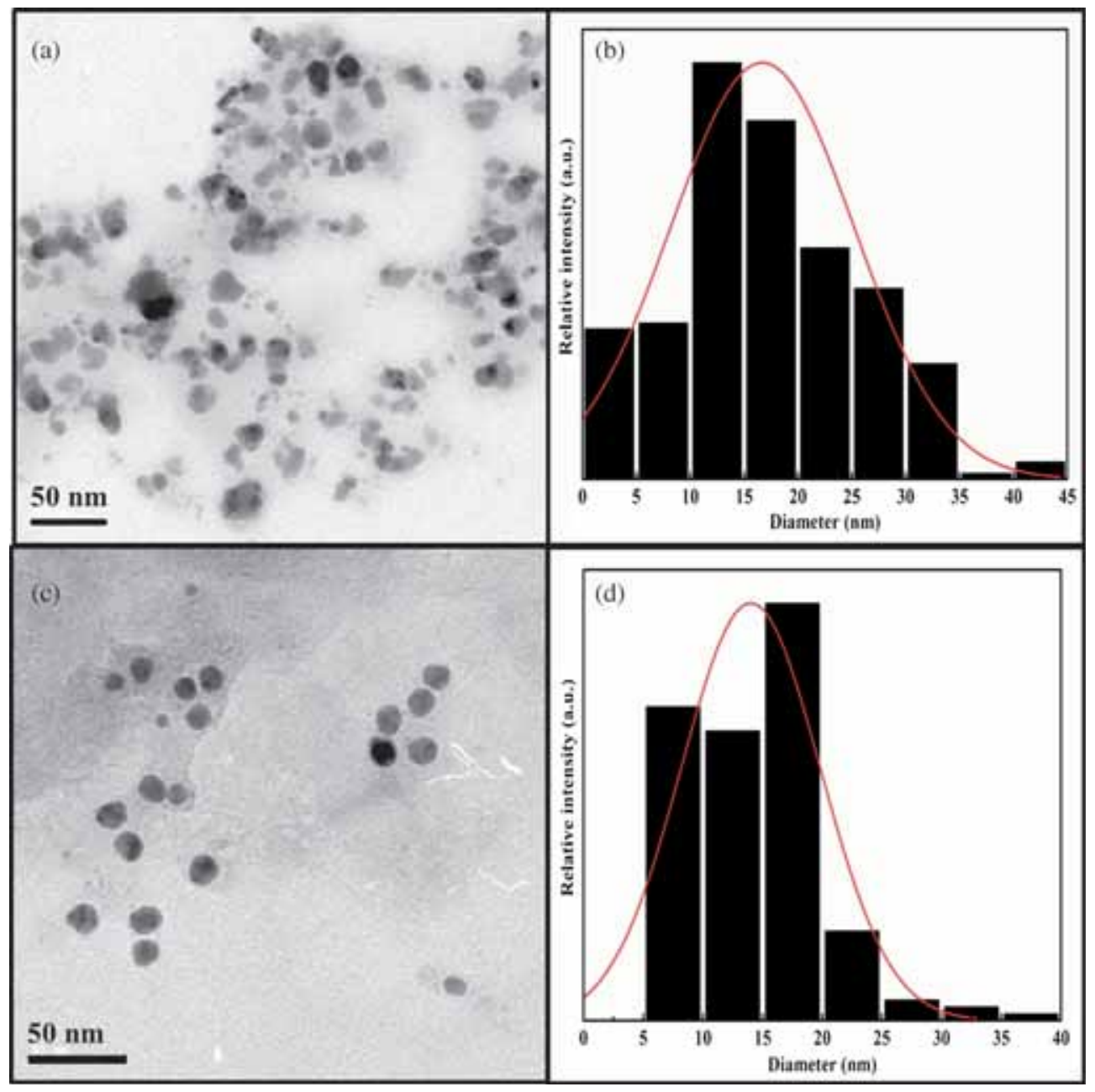

Figure 3. TEM image of samples S1 and S2 of c-AgNPs (a, c), and the corresponding size distributions (b, d), respectively. 


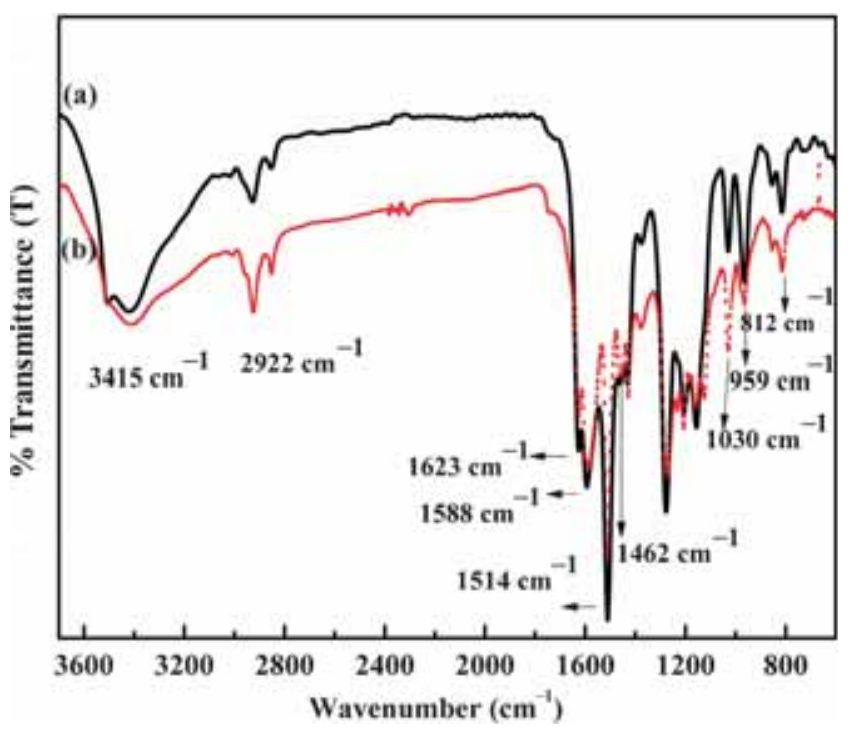

Figure 4. FT-IR spectra of (a) pure curcumin and (b) curcumin stabilized AgNPs (sample S2).

\section{2 c-AgNPs catalyzed p-nitrophenol (Nip) reduction}

The UV-Visible spectrum of the Nip solution exhibited a strong absorption peak at $317 \mathrm{~nm}$. On addition of $\mathrm{NaBH}_{4}$ to the Nip solution, the absorbance peak shifted to $401 \mathrm{~nm}$ due to conversion of Nip to $p$-nitrophenolate ion. Then, the UV-Visible spectrum of a blank experiment of Nip reduction by $\mathrm{NaBH}_{4}$ (without c-AgNPs) was recorded (Figure 5a). In this case, the peak absorbance at $401 \mathrm{~nm}$ showed no change with time confirming that there is no Nip reduction in the absence of c-AgNPs. Next, the reaction kinetics was studied after the addition of c-AgNPs. Figure 5b displays decrease in the intensity of the absorption peak at $401 \mathrm{~nm}$ after addition of c-AgNPs with progress of time. Simultaneously, a smaller absorbance peak at $300 \mathrm{~nm}$ emerged and gradually increased due to the formation of the reaction product $p$-aminophenol (AP). Also, the spectrum showed two isosbestic points at 280 and $312 \mathrm{~nm}$ which indicate that Nip was fully converted to AP without any side reaction. We observed that on completion of the reaction, the absorption peak originally at $401 \mathrm{~nm}$ undergoes slight blue shift and also its intensity did not go to zero. The remaining absorbance at this slightly blue shifted maximum is due to the LSPR of the c-AgNPs since the intensity of this absorbance was the same as that of the aqueous dispersion of c-AgNPs used (shown in Figure 5b). As is well-known, the blue shift of the LSPR absorbance is due to the adsorption of phenolate ions on the surface of the silver nanoparticles. ${ }^{26,27}$

Monitoring of the absorbance of $p$-nitrophenolate ion showed that it initially did not change for some interval of time. The time during which no reduction took place is known as induction time $\left(t_{\text {ind }}\right)$ and it was observed that the reaction started only after the elapse of $t_{\text {ind }}$. For both samples S1 and S2 of c-AgNPs, after the elapse of $t_{\text {ind }}$, linear plot of $\ln \left(A / A_{0}\right)$ versus time was obtained. Here, $A$ and $A_{0}$ denote the absorbance of $p$-nitrophenolate ion at time $t$ and $t_{0}$, respectively. The reaction, therefore, followed pseudo first-order kinetics since the concentration of $\mathrm{NaBH}_{4}$ (taken in large excess relative to Nip) was virtually constant throughout the reaction. These observations are in agreement with previously published data in literature on $\mathrm{Ag}$ nanoparticle
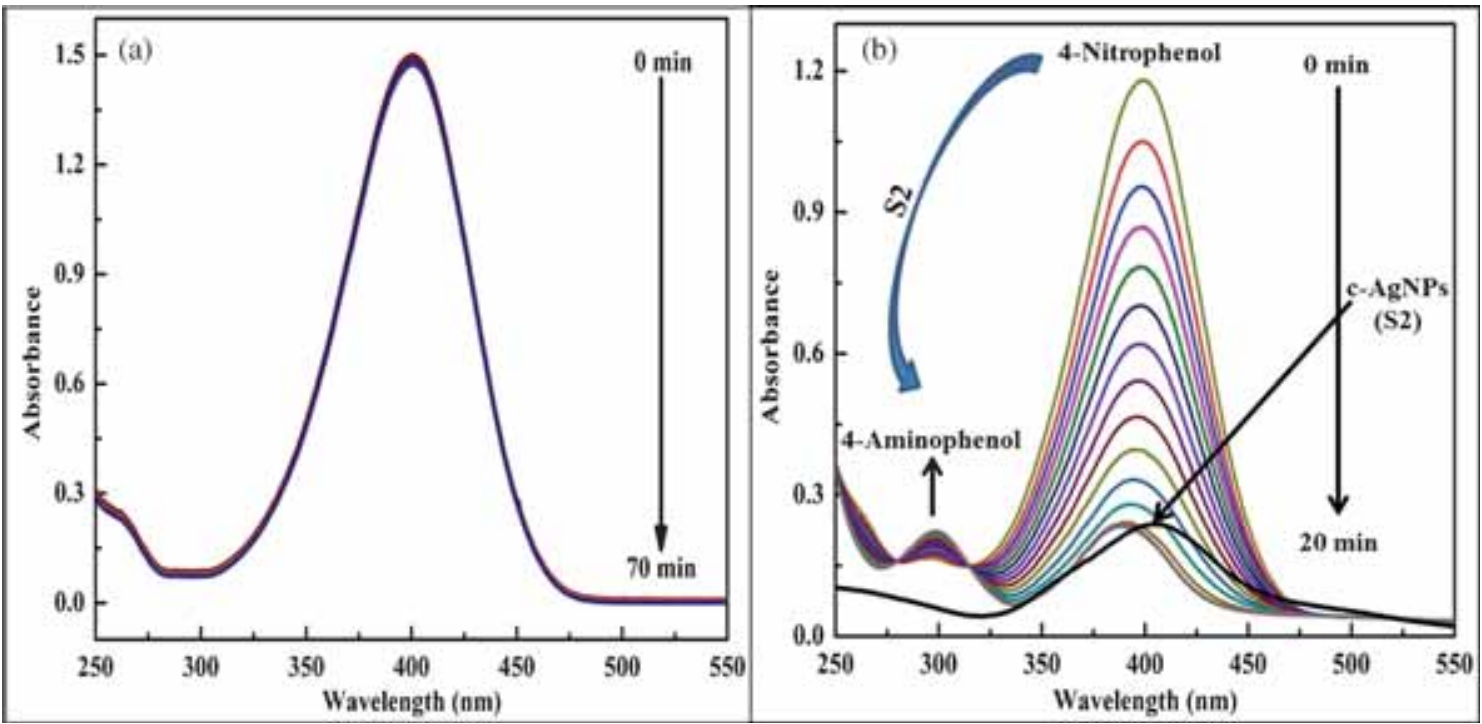

Figure 5. UV-VIS absorption spectraof $p$-nitrophenol and $\mathrm{NaBH}_{4}$ versus time of (a) the blank sample (without c-AgNPs catalyst) and (b) in presence of c-AgNPs (sample S2) catalyst. 
catalyzed reduction of Nip. ${ }^{28,29}$ The rate expression for this reaction is given by the equation (1).

$$
\frac{d[N i p]}{d t}=-k_{a p p}[N i p]=-k_{1} S[N i p]
$$

Here, [Nip] is the concentration of $p$-nitrophenol, while $k_{1}$ is the surface area normalized rate constant. Surface area $(S)$ of the nanoparticles was calculated by using average radius obtained by TEM micrographs and their total mass per particle. The bulk density of the silver nanoparticle was taken into consideration as 10.49 $\mathrm{g} / \mathrm{cm}^{3}$. The rate constant $\left(k_{a p p}\right)$ was determined in a straightforward manner from the slope of the linear fit of $\ln \left(A / A_{0}\right)$ with time $t$ (after excluding $t_{\text {ind }}$ as shown in Figure 6a). We observe that the $k_{a p p}$ for sample S2 is much greater than S1. Owing to the finer particle size as well as narrower size distribution, sample S2 has a higher surface area than the c-AgNPs in sample S1. The difference in $k_{a p p}$ may therefore be either due to the increased surface area of the S2 catalyst or due to activation energy of catalytic reaction or both. To find the activation energies involved, rate constants at different temperatures were analyzed in accordance with the Arrhenius equation. The linearized form of Arrhenius equation is given below.

$$
\ln k=\ln A-E_{\mathrm{a}} / R T
$$

The activation energy $\left(E_{\mathrm{a}}\right)$ was calculated from the slope $\left(-E_{\mathrm{a}} / R\right)$ of the linear fit of the $\ln k$ versus $1 / \mathrm{T}$ plot which presented in Figure $6 \mathrm{~b}$. The activation energy $E_{\mathrm{a}}$ when the reaction is catalyzed by $\mathrm{S} 1$ was found to be $\sim 94 \mathrm{~kJ} \mathrm{~mol}^{-1}$ and $\sim 64 \mathrm{~kJ} \mathrm{~mol}^{-1}$ when catalyzed by S2. We may, therefore, conclude that the much greater $k_{a p p}$ for sample S2 is due to a combination of increased sur- face area as well as lower activation energy. It is worthwhile to note that the activation energy value reported for sample S2 in the present study is comparable to those reported earlier for catalytic p-nitrophenol reduction. ${ }^{30-34}$ As mentioned earlier, this reaction occurs on the surface of catalyst nanoparticles as per the $\mathrm{LH}$ model. In the LH mechanism, the two reactants adsorb onto adjacent active sites. The reactants then react and product thus formed desorbs leaving behind empty active sites on the catalyst surface. This model, therefore, requires that the reaction between the adsorbed species on the catalyst should be the rate determining step which is well-established for the nanoparticle catalyzed Nip reduction reactions. On the other hand, the adsorption/desorption equilibration is assumed to be fast. In view of the aforementioned assumptions, the rate constant is given by equation (1). ${ }^{19,20}$

$$
k_{\text {app }}=\frac{k S K_{N i p}^{n}[N i p]^{n-1}\left(K_{B H_{4}}\left[B H_{4}\right]\right)^{m}}{\left(1+\left(K_{N i p}[N i p]\right)^{n}+\left(K_{B H_{4}}\left[B H_{4}\right]\right)^{m}\right)^{2}}
$$

In the above equation, $k$ is the molar rate constant per square meter of the catalyst surface or the true rate constant related to the surface reactivity of the adsorbed species,,$^{20,24}$ is the total surface area of metal nanoparticles normalized to the unit volume of the solution, $K_{N i p}$ is the equilibrium adsorption constant of Nip, $K_{B H_{4}}$ is the equilibrium adsorption constant of borohydride, while [Nip] and $\left[\mathrm{BH}_{4}\right]$ are the concentrations of Nip and borohydride, respectively. Exponent $\mathrm{n}$ and $\mathrm{m}$ are indicative of the heterogeneity of adsorbent active sites. Thus, while the value of 1 represents the classical Langmuir adsorption isotherm, lower values indicate increasing heterogeneity.
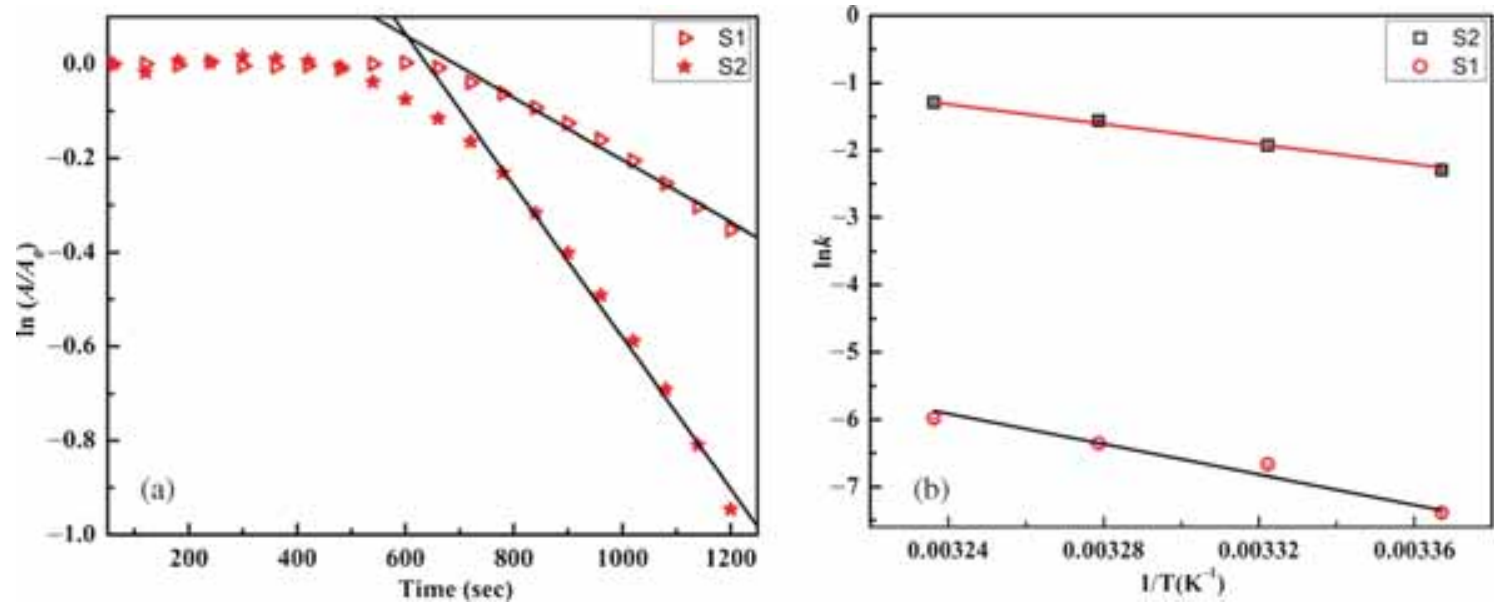

Figure 6. (a) Variation of $\ln (\mathrm{A} / \mathrm{A} 0)$ [absorbance (A) was measured at $\lambda=401 \mathrm{~nm}$ ] versus time for $\mathrm{S} 1$ and S2 samples of c-AgNPs. (b) Arrhenius plots for Nip reduction reaction catalyzed by S1 and S2 samples of c-AgNPs (Error bars cannot be seen because in most cases they are smaller than the symbol size). 

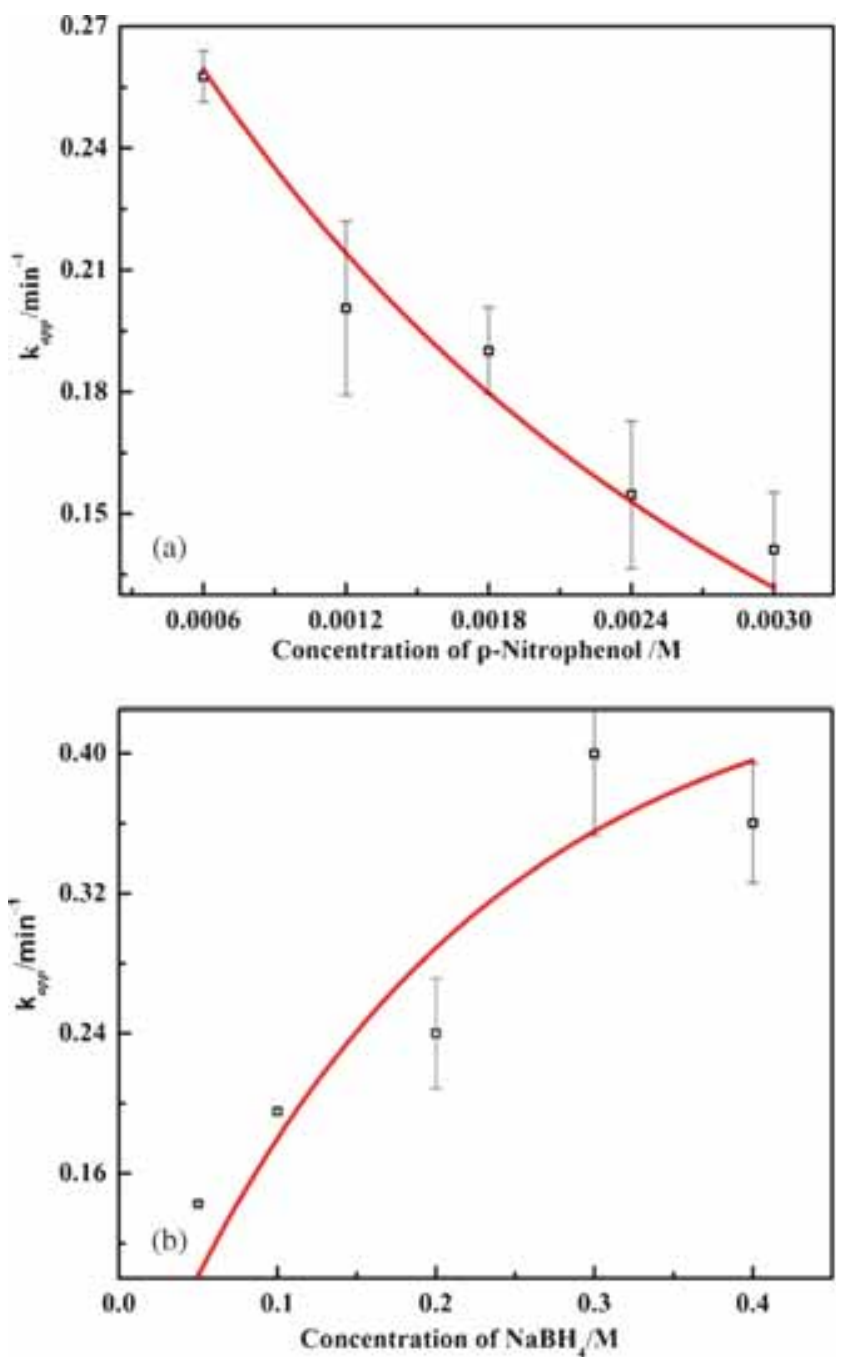

Figure 7. (a) Variation of $k$ as a function of different initial concentrations of $\operatorname{Nip}\left(0.6,1.2,1.8,2+-.4,3.0 \times 10^{-3} \mathrm{M}\right)$, at $\left[\mathrm{NaBH}_{4}\right]=0.1 \mathrm{M}$, mass (sample S2 c-AgNPs) $=0.004 \mathrm{mg}$. (b) $\left[\mathrm{NaBH}_{4}\right](0.05,0.1,0.2,0.3,0.4 \mathrm{M})$ at $[\mathrm{Nip}]=1.2 \times$ $10^{-3} \mathrm{M}$ and mass (sample S2 c-AgNPs) $=0.004 \mathrm{mg}$. The solid line shows the fit of LH model (eq. 3) with the experimental data. The surface area of c-AgNPs is $3.79 \mathrm{~m}^{2} \mathrm{~L}^{-1}$.

The experimental data required for fitting with equation 3 were obtained by conducting kinetics experiments by investigating the effect of reactant concentration on the rate of reduction. These experiments were carried out at the constant temperature at $301 \mathrm{~K}$, only on sample S2 as the catalyst in view of its much better catalytic activity. Figures $7 \mathrm{a}$ and $7 \mathrm{~b}$ show the effect of concentration variation of $\mathrm{Nip}$ and $\mathrm{NaBH}_{4}$ on $k_{a p p}$. Figure $7 \mathrm{a}$ displays the decrease in $k_{a p p}$ with increase in the Nip concentration. On the other hand, $k_{a p p}$ increased with the concentration of $\mathrm{NaBH}_{4}$ on going from 0.05 to $0.3 \mathrm{M}$, but on further increasing the $\mathrm{NaBH}_{4}$ concentration (i.e., $0.4 \mathrm{M}$ ) slight decrease in $k_{\text {app }}$ was observed (Figure 7b). It appears that at higher concentration of $\mathrm{NaBH}_{4}$, the rate constant reaches a saturation value. These observations are as per the Langmuir-Hinshelwood mechanism (LH) model. ${ }^{24,35}$ The solid lines in the figures are fits to the experimental data as per equation (1). To obtain the solid line fits, the parameters were optimised until the best fit was found such that both figures (7a and $7 \mathrm{~b}$ ) could be fitted with same set of values and errors involved were the minimum. Table 1 gives these Langmuir-Hinshelwood parameters for the present catalytic reduction and also compares them with those available in literature. Now, we discuss the observations in the context of the above-mentioned LH mechanism. In this context, it is important to note that large $\left[\mathrm{NaBH}_{4}\right] /[\mathrm{Nip}]$ ratio was used and the fact that increase in [Nip] led to decrease in $k_{a p p}$. This is explained by the fact that the adsorption constant of Nip is much larger than that of $\mathrm{NaBH}_{4}$ (Table 1). As seen from Table 1, other authors have also found that this is typically the case. Owing to the larger adsorption constant of Nip on nanoparticle surface, increasing its concentration results in blockage of active sites on the catalyst surface. Thus, at some Nip concentration, nearly full coverage of the catalyst is possible. This would be manifested by very low catalytic activity, ${ }^{36}$ as happens in our observations as well. Combined information pertaining to respective adsorption constants of the reactants with LH mechanism implies that in the present system competition between reactants for active sites on the catalyst surface slows down the reaction. An optimum

Table 1. Comparison of surface rate constants, $\mathrm{k}$, and adsorption constants of both Nip and $\mathrm{BH}^{-}\left(\mathrm{K}_{\mathrm{Nip}}\right.$ and $\left.\mathrm{K}_{\mathrm{BH} 4-}\right)$ obtained by fitting the experimentally determined $k_{a p p}$ to the LH equation (1).

\begin{tabular}{lcccccc}
\hline Catalyst & $\mathrm{k} \times 10^{-6}\left(\mathrm{~mol} . \mathrm{m}^{-2} \cdot \mathrm{s}^{-1}\right)$ & $\mathrm{K}_{\mathrm{Nip}}(\mathrm{L} / \mathrm{mol})$ & $\mathrm{K}_{\mathrm{BH} 4}^{-}(\mathrm{L} / \mathrm{mol})$ & $\mathrm{n}$ & $\mathrm{m}$ & Reference \\
\hline $\mathrm{Pt}$ & $460 \pm 60$ & $2300 \pm 500$ & $89 \pm 10$ & $0.6 \pm 0.1$ & $1 \pm 0.1$ & 19 \\
$\mathrm{Au}$ & $160 \pm 60$ & $5500 \pm 1000$ & $58 \pm 5$ & $0.6 \pm 0.1$ & $1 \pm 0.1$ & 19 \\
$\mathrm{Pd}$ & $55 \pm 50$ & $2300 \pm 400$ & $48 \pm 5$ & 0.6 & 1 & 20 \\
$\mathrm{Au}_{75} \mathrm{Pd}_{25}$ & $2900 \pm 300$ & $1450 \pm 300$ & $122 \pm 15$ & 0.6 & 1 & 20 \\
G4 RuDENs & $18 \pm 3.9$ & $90 \pm 6$ & $3.6 \pm 1.1$ & $0.37 \pm 0.1$ & $0.85 \pm 0.08$ & 21 \\
G5 RuDENs & $24 \pm 6$ & $87 \pm 4$ & $5.4 \pm 1.1$ & $0.56 \pm 0.1$ & $0.57 \pm 0.11$ & 21 \\
$\mathrm{Au}$ & $730 \pm 100$ & $5300 \pm 800$ & $79 \pm 12$ & $0.6 \pm 0.1$ & $1 \pm 0.1$ & 24 \\
$\mathrm{c}-$ AgNPs & $3190 \pm 72$ & $217.92 \pm 7.36$ & $1.65 \pm 0.23$ & 1 & 1 & This work \\
\hline
\end{tabular}


Table 2. Comparison of apparent reaction rate constant and turnover frequencies (TOF) for $p$-nitrophenol reduction.

\begin{tabular}{lcccc}
\hline Sr. No. & Catalyst & $\begin{array}{c}\text { Apparent reaction rate } \\
\text { constant }\left(k_{\text {app }}\right)\left(\mathrm{s}^{-1}\right)\end{array}$ & $\begin{array}{c}\text { Turnover Frequency } \\
(\mathrm{TOF})\left(\mathrm{min}^{-1}\right)\end{array}$ & Reference \\
\hline 1 & CuNPs & $1.588 \times 10^{-3}$ & 0.0122 & 28 \\
2 & Au/Graphene hydrogel & $3.17 \times 10^{-3}$ & 68.36 & 37 \\
3 & Au on PMMA & $7.9 \times 10^{-3}$ & 0.506 & 38 \\
4 & Au NCs & $2.1 \times 10^{-3}$ & 0.264 & 39 \\
5 & Au on Carbon nanotubes & $1.10 \times 10^{-4}$ & 0.116 & 40 \\
6 & AgNPs & $3.28 \times 10^{-3}$ & 40.2 & 41 \\
7 & AgNP/PANINF & $21.39 \times 10^{-3}$ & 201.5 & 42 \\
8 & AgNP-PG-5K & $5.50 \times 10^{-3}$ & 320 & 43 \\
9 & Au-MgO & $7.6 \times 10^{-3}$ & 0.487 & This work \\
10 & CuNPs & $4.3 \times 10^{-3}$ & 671 & This work \\
11 & c-AgNPs (S1) & $6.60 \times 10^{-4}$ & 1081 & 1600 \\
12 & c-AgNPs (S2) & $6.66 \times 10^{-3}$ & &
\end{tabular}

concentration of Nip and $\mathrm{NaBH}_{4}$ is therefore required for the achieving the maximum reaction rate. Both $\mathrm{m}$ and $\mathrm{n}$ exponent values found from our fits are equal to 1 which agree with the Langmuir isotherm or uniform adsorption sites.

As is evident from the above description of equation (1), for catalytic systems which follow the LH mechanism, the true or the surface rate constant $k$ is the most appropriate quantity to compare the catalytic activities of various catalysts. One can see that among the catalysts for which such data is available in literature (Table 1), the present c-AgNPs catalyst has the best $k$ value. On the other hand, generally in most publications, the catalytic activities of the different nanocatalysts are compared on the basis of their apparent rate constants. However, in this approach the influence of the amount of the catalyst is ignored. Therefore, in Table 2, we present a comparison of the turnover frequency (TOF) of c-AgNPs with values obtained in some recent studies. The turnover frequency (TOF) was calculated using the following expression.

$$
T O F=\frac{\text { Number of moles of reactant }}{\text { Number of moles of catalyst }} \text { yield/Time }
$$

From the Table we see that the TOF obtained for c-AgNPs are better than other systems reported in literature. Earlier Chang et al., and Baruah et al., reported the use of Ag nanoparticles of comparable size as catalysts for Nip reduction. The TOF values calculated from their publications are much lower than those found for c-AgNPs in the present investigation. ${ }^{41,42} \mathrm{~A}$ possible reason could be that curcumin on being attached to the metal acts in some synergistic way (with respect to the catalyst being an electron conduit for Nip reduction) to increase number of catalytic active sites per unit surface area of the catalyst. ${ }^{45}$ The extended conjugated electronic structure of curcumin means that the $\pi$ - electron cloud is delocalized over the entire molecule. It is possible that this feature of curcumin may be related to the enhancement of catalytic activity of c-AgNPs.

\section{Conclusions}

Two different size distributions of fine curcumin stabilized silver nanoparticles have been synthesized using a sonochemical protocol. Nanoparticle size distribution became narrower and average sizes finer 'on increasing the ratio of precursor silver salt to curcumin. The catalytic activities of these nanoparticles were investigated on the $p$-nitrophenol reduction model reaction. Silver nanoparticles with finer size distribution (sample S2) demonstrated significantly better catalytic activity parameters. However, the activation energy as per the Arrhenius equation for the reaction catalyzed by $\mathrm{S} 2$ was also lower than that for sample S1. Thus, better catalytic activity of the reaction catalyzed by sample $\mathrm{S} 2$ was because of the lower activation energy as well as increased catalyst surface area. It is important to note that curcumin stabilization of the AgNPs significantly enhances the turnover frequency value and the value obtained (for S2) is better than those reported in literature till date for $p$-nitrophenol reduction. Also, the analysis of the effect of reactant concentration on the rate constant shows that the reduction of $p$-nitrophenol by borohydride in presence of curcumin stabilized silver nanoparticles occurs by the Langmuir-Hinshelwood mechanism. Using this model we were able to calculate the surface reactivity of the adsorbed species given by the kinetic constant $k$, and also the thermodynamic adsorption constants $K_{N i p}$ and $K_{B H_{4}}$ which are used to understand the mechanism better. 


\section{Acknowledgements}

ADV acknowledges financial support received from UGC in form of SRF. TEM images were recorded at the Electron microscope facility of Department of Metallurgical Engineering IIT(BHU). IS and ADV thank Prof. A. K. Mukherjee for use of Origin 2015 software.

\section{References}

1. Ghosh S K and Pal T 2007 Chem. Rev. 1074797

2. Lee H Y, Li Z, Chen K, Hsu A R, Xu C, Xie J, Sun S and Chen X 2008 J. Nucl. Med. 491371

3. Zeng J, Huang J, Lu W, Wang X, Wang B, Zhang S and Hou J 2007 Adv. Mater. 192172

4. Tan M, Wang G, Ye Z and Yuan J 2006 J. Lumin. 11720

5. Lee J S, Ulmann P A, Han M S and Mirkin C A 2008 Nano Lett. 8529

6. Pissuwan D, Valenzuela S M and Cortie M B 2006 Trends Biotechnol. 2462

7. Panigrahi S, Kundu S, Ghosh S, Nath S and Pal T 2004 J. Nanopart. Res. 6411

8. Iravan S 2011 Green Chem. 132638

9. Song J Y and Kim B S 2009 Bioprocess Biosyst. Eng. 3279

10. Georgiev V, Ananga A and Tsolova V 2014 Nutrient 6391

11. Dwyer K, Hosseinian F and Rod M 2014 J. Food Res. 391

12. Sindhu K, Rajaram A, Sreeram K J and Rajaram R 2014 RSC Adv. 41808

13. Sreelakshmi C, Goel N, Datta K K R, Addlagatta A, Ummanni R and Reddy B V S 2013 Nanosci. Nanotechnol. Lett. 51

14. Bettini S, Pagano R, Valli L and Giancane G 2014 Nanoscale 610113

15. Patil D, Nag S, Basak A and Nag A 2008 Inter. J. Chem. Sci. 611

16. Venkatesham M, Ayodhya D, Madhusudhan A, Babu N V and Veerabhadram G 2014 Appl. Nanosci. 4113

17. Joseph S and Mathew B 2014 Res. J. Recent Sci. 3185

18. Bang B J H and Suslick K S 2010 Adv. Mater. 221039

19. Wunder S, Polzer F, Lu Y, Mei Y and Ballauff M 2010 J. Phys. Chem. C 1148814

20. Kaiser J, Leppert L, Welz H, Polzer F, Wunder S, Wanderka N, Albrecht $\mathrm{M}$, Lunkenbein $\mathrm{T}$, Breu J,
Kmmel S, Lu Y and Ballauff M 2012 Phys. Chem. Chem. Phys. 146487

21. Antonels N C and Meijboom R 2013 Langmuir 2913433

22. Nemanashi M and Meijboom R 2013 J. Colloid Inter. Sci. 389260

23. Bingwa N and Meijboom R 2014 J. Phys. Chem. C 118 19849

24. Wunder S, Lu Y, Albrecht M and Ballauff M 2011 ACS Catal. 1908

25. Vasileva P, Donkova B, Karadjova I and Dushkin C 2011 Colloid Surf. A 382203

26. Pradhan N, Pal A and Pal T 2001 Langmuir 171800

27. Henglein A 1989 Chem. Rev. 891861

28. Deka P, Deka R C and Bharali P 2014 New J. Chem. 381789

29. Yang X, Zhong H, Zhu Y, Jiang H, Shen J, Huang J and Li C 2014 J. Mater. Chem. A 29040

30. Zhu M, Wang C, Menga D and Diao G 2013 J. Mater. Chem. A 12118

31. Lu Y, Mei Y, Walker R, Ballauff M and Drechsler M 2006 Polymer 474985

32. Shin K S, Choi J, Park C S, Jang H J and Kim K 2009 Catal. Lett. 1331

33. Pradhan N, Pal A and Pal T 2002 Colloid Surf. A 196247

34. Saha S, Pal A, Kundu S, Basu S and Pal T 2010 Langmuir 262885

35. Herves P, Lorenzo M P, Marzan L M L, Dzubiella J, Lu Y and Ballauff M 2012 Chem. Soc. Rev. 415577

36. Lin C, Tao K, Hua D, Ma Z and Zhou S 2013 Molecules 1812609

37. Li J, Liu C and Liua Y 2012 J. Mater. Chem. 228426

38. Kuroda K, Ishida T and Haruta M 2009 J. Mol. Catal. A-Chem. 2987

39. Rashid M H, Bhattacharjee R R, Kotal A and Mandal T K 2006 Langmuir 227141

40. Abdel-Fattah M and Wixtrom T A 2014 ECS J. Solid State Sci. Tech. 3 M18

41. Chang G, Luo Y, Lu W, Qin X, Asiri A, MAl-Youbi A O and Sun X 2012 Catal. Sci. Technol. 2800

42. Baruah B, Gabriel G J, Akbashev M J and Booher M E 2013 Langmuir 294225

43. Layek K, Kantam M, Shirai M, Nishio-Hamane D, Sasaki T and Maheswaran H 2012 Green Chem. 143164

44. Verma A D, Mandal R K and Sinha I 2015 Catal. Lett. 1451885

45. Priyadarsini K 2014 Molecules 1920091 